\title{
REVIEWS
}

\section{Yoga for Treating Headaches: a Systematic Review and Meta-analysis}

\author{
Dennis Anheyer, $M A^{7}$ (D), Petra Klose, $P h D^{7}$, Romy Lauche, $P h D^{2,3}$, \\ Felix Joyonto Saha, $\mathrm{MD}^{7}$, and Holger Cramer, $P h D^{1,3}$ \\ 'Department of Internal and Integrative Medicine, Kliniken Essen-Mitte, Faculty of Medicine, University of Duisburg-Essen, Essen, Germany; \\ ${ }^{2}$ Department of Internal and Integrative Medicine, Sozialstiftung Bamberg, Bamberg, Germany; ${ }^{3}$ Australian Research Centre in Complementary \\ and Integrative Medicine (ARCCIM), Faculty of Health, University of Technology Sydney, Sydney, New South Wales, Australia.
}

BACKGROUND: Headache disorders are currently the sixth leading cause of disability across the globe and therefore carry a significant disease burden. This systematic review and meta-analysis aims to investigate the effects of yoga on headache disorders.

METHODS: MEDLINE/PubMed, Scopus, the Cochrane Library, and PsycINFO were screened through May 2019. Randomized controlled trials (RCTs) were included when they assessed the effects of yoga in patients with a diagnosis of chronic or episodic headache (tensiontype headache and/or migraine). Usual care (no specific treatment) or any active treatments were acceptable as control interventions. Primary outcome measures were headache frequency, headache duration, and pain intensity. For each outcome, standardized mean differences (SMD) and 95\% confidence intervals (CI) were calculated. RESULTS: Meta-analysis revealed a statistically significant overall effect in favor of yoga for headache frequency (5 RCTs; standardized mean difference $(\mathrm{SMD})=-1.97$; $95 \%$ confidence interval (CI) -2.75 to $-1.20 ; I^{2}=63.0 \%$, $\tau^{2}=0.25, P=0.03$ ), headache duration (4 RCTs; SMD $=-$ 1.45; $95 \%$ CI -2.54 to $-0.37 ; I^{2}=69.0 \%, \tau^{2}=0.33, P=$ 0.02 ), and pain intensity (5 RCTs; SMD $=-3.43 ; 95 \% \mathrm{CI}-$ 6.08 to $\left.-0.70, I^{2}=95.0 \%, \tau^{2}=4.25, P<0.01\right)$. The significant overall effect was mainly due to patients with tension-type headaches. For patients with migraine, no statistically significant effect was observed.

DISCUSSION: Despite discussed limitations, this review found preliminary evidence of short-term efficacy of yoga in improving headache frequency, headache duration, and pain intensity in patients suffering from tensiontype headaches. Further studies are urgently needed to draw deeper conclusions from the available results.

KEY WORDS: yoga; complementary therapies; tension-type headache; migraine; meta-analysis; review.

$\mathrm{J}$ Gen Intern Med 35(3):846-54

DOI: $10.1007 / \mathrm{s} 11606-019-05413-9$

(c) Society of General Internal Medicine 2019

\section{BACKGROUND}

Chronic headache is characterized by the presence of pain in the region of the head for at least fifteen days per month for

Received June 17, 2019

Accepted September 20, 2019

Published online October 30, 2019 more than 3 months. ${ }^{1}$ Among others, chronic headache includes chronic migraine, chronic tension-type headache (TTH), and chronic cluster headache. The mean global prevalence rate of all headaches is $47 \%$, while TTH and migraine have a prevalence of $38 \%$ and $10 \%$, respectively. ${ }^{2}$ In the USA, migraine alone contributes to 86.5 million lost workdays each year and to an indirect annual cost of US $\$ 9.3$ billion. ${ }^{3}$ Being the sixth leading cause of disability worldwide, ${ }^{4}$ headache is a major public health problem, strongly limiting quality of life. ${ }^{5}$ Patients with chronic headache even report significantly lower quality of life than those with episodic headache. ${ }^{5}$

Development and maintenance of headache is associated with modifiable and non-modifiable risk factors, including stress, obesity, medication overuse, psychiatric comorbidities, dietary, and sleep patterns. ${ }^{6}$ While headache treatment is mainly focused on medicinal symptom management, ${ }^{7}$ patients generally view but focusing on the cause of the headache as a priority. ${ }^{8} \mathrm{~A}$ further problem with medicinal headache management is the risk of serious harms including medication overuse headache, gastrointestinal bleeding (non-steroidal antiinflammatory drugs), liver damage (acetaminophen), addiction (opioids), memory impairment, depression (topiramate), weight gain, and precipitation of cardiac arrhythmias and seizures (amitriptyline). ${ }^{9}$

In light of these concerns safe, effective nonpharmacological treatments focusing on the cause of chronic and episodic headache are needed. One such approach could be to target chronic stress, which plays a major role in the development, maintenance, and chronification of headache. ${ }^{10}$ It is hypothesized that stress can induce nociceptor sensitization and increase other risk factors of headache, such as sleep impairment, obesity, and psychopathology. ${ }^{10}$ Thus, stress reduction might be an important treatment strategy for headache, and there is an emerging evidence base to support this view. ${ }^{11}$

An important non-pharmacological treatment approach that has been shown to effectively reduce chronic pain, ${ }^{12,13}$ stress, ${ }^{14}$ and several other headache risk factors such as psychiatric conditions ${ }^{15-17}$ and obesity ${ }^{18}$ is yoga. Yoga has been used for health reasons by an estimated 31 million American adults at least once in their lifetime, with pain and stress relief being among the most common reasons for this use. ${ }^{19}$ 
Deriving from traditional Indian philosophy, yoga comprises physical postures as well as advice for ethical lifestyle and spiritual practice with the ultimate goal of uniting mind, body, and spirit. ${ }^{20,21}$ In North America and Europe, yoga is most often associated with physical postures (asana), breathing techniques (pranayama), and meditation (dhyana). ${ }^{20}$ A variety of yoga schools have evolved from the traditional Indian system of yoga in Western societies, which are giving different weight to physical and spiritual practice. ${ }^{20}$ Although the use of yoga for headache appears reasonable, no systematic review and meta-analysis so far has assessed its efficacy and safety in this condition.

This systematic review and meta-analysis aims to address this knowledge gap. This review systematically assesses the efficacy and safety of yoga in reducing the frequency, duration, and/or intensity of headache in patients with chronic or episodic headache.

\section{METHODS}

This review was conducted in accordance with the Preferred Reporting Items for Systematic Reviews and Meta-Analyses (PRISMA) guidelines and the Cochrane handbook for systematic reviews of interventions. ${ }^{22,23}$ A protocol of this review was not prospectively registered.

\section{Eligibility Criteria}

Patients. Studies of patients with a diagnosis (in accordance to the ICHD-III criteria) of episodic or chronic tension-type headache and/or migraine with or without aura were included in this review. No other headache type was considered to be eligible. No restrictions regarding age or gender were made.

Intervention. Studies that assessed yoga as the main intervention were included. No restrictions regarding yoga style or length of the intervention period were applied. Usual care (no specific treatment) or any active comparator were acceptable as control interventions.

Outcomes. Studies were included if they assessed at least (1) headache frequency, (2) headache duration, or (3) pain intensity as primary outcome measures.

Study Type. Only randomized controlled trials (RCTs) were included while observational studies or non-randomized trials were excluded. Studies were included only if they were published as full-text articles in peer-reviewed scientific journals and in English or German language.

The review was limited to RCTs as they represent the highest level of primary research evidence for clinical interventions. Further, RCTs are designed to control for both known and unknown confounders and to establish a clear causal link between treatment and outcome. ${ }^{20}$

\section{Literature Search}

The literature search comprised the following electronic databases from their inception through 04 May 2019: MEDLINE/ PubMed, Scopus, the Cochrane Library, and PsycINFO. Embase was not searched separately since it is partly included in Scopus. The complete search strategy for MEDLINE was as follows: ("Yoga"[Mesh] OR yoga[Title/Abstract] OR yogic[Title/Abstract] OR asana[Title/Abstract] OR pranayama[Title/Abstract] OR dhyana[Title/Abstract]) AND (Tension-Type Headache[MeSH Terms] OR Tension-Type Headache[Title/Abstract] OR TTH[Title/Abstract] OR Migraine with Aura[MeSH Terms] OR Migraine without Aura[MeSH Terms] OR migraine*[Title/Abstract]). The search strategy was adapted for each database as necessary. In addition, reference lists of identified original articles or reviews were searched manually. Databases for grey literature were not searched.

The literature search was performed by DA. Identified abstracts were screened independently by two review authors (DA, PK). Potentially eligible articles were also read in full text by two authors (DA, PK), independently. Discrepancies were rechecked and, if necessary, discussed with a third reviewer $(\mathrm{HC})$ until consensus was achieved.

\section{Data Extraction}

Two reviewers (DA, HC) independently extracted data on characteristics of the study (e.g., trial design, randomization, blinding), characteristics of the patient population (e.g., sample size, age, diagnosis), characteristics of the intervention, and control condition (e.g., type, program length, frequency, and duration), drop-outs, outcome measures, follow-ups, results, and safety. Discrepancies were rechecked and discussed with a third reviewer (PK) until consensus was achieved.

Risk of Bias in Individual Studies. Risk of bias was assessed by reviewers (DA, HC) independently using the Cochrane risk of bias tool. This tool assesses risk of bias on the following domains: selection bias, performance bias, detection bias, attrition bias, reporting bias, and other bias. ${ }^{23}$ Discrepancies were rechecked with a third reviewer (PK) and consensus achieved by discussion. If necessary, trial authors were contacted for further details.

Quality of Evidence. To take the quality of the respective studies as well as the confidence in the results into account, the quality of evidence and the strength of recommendation was graded by to reviewers independently (DA and PK). Discrepancies were rechecked with a third reviewer (HC) and consensus achieved by discussion. The recommendations of the Grading of Recommendations Assessment, Development and Evaluation (GRADE) working group were used. The quality of evidence was graded as high quality, moderate quality, low quality, or very low quality based on the limitations of the respective studies, the inconsistency between the results of the respective, the indirectness of the evidence, the 
imprecision of the findings, the risk of publication bias, and the risk of other bias. Importantly, GRADE does not evaluate the quality of single studies but the quality of the complete body of evidence; e.g., the evidence for effects of yoga compared to a specific comparator on headache symptoms is rated.

\section{Data Analysis}

Assessment of Overall Effect Size. Random effects metaanalysis estimates were obtained using $\mathrm{R}$ software Version 3.5.3 (R Foundation for Statistical Computing, Vienna, Austria. URL https://cran.r-project.org) and the packages "metafor" (written by Wolfgang Viechtbauer; http://www. metafor-project.org/) and "meta" (written by Guido Schwarzer; https://CRAN.R-project.org/package=meta). The Knapp-Hartung small-sample correction was used because it provides a more adequate accounting of uncertainty when pooling treatment effects from a small number of heterogeneous studies. ${ }^{24-27}$ Standardized mean differences (SMD) with $95 \%$ confidence intervals (CI) were calculated as the difference in means between groups divided by the pooled standard deviation using Hedges' correction for small study samples. ${ }^{23}$ Where no standard deviations were available, they were calculated from standard errors, confidence intervals, or $t$ values ${ }^{23}$ or attempts were made to obtain the missing data from the trial authors by email. For all observed outcome measures, a negative SMD indicated beneficial effects for the yoga group, compared to the comparison group.

Cohen's categories were used to evaluate the magnitude of the overall effect size with $\mathrm{SMD}=0.2-0.5$, small; $\mathrm{SMD}=0.5$ 0.8 , medium; and SMD $>0.8$, large effect sizes. ${ }^{28}$

Assessment of Heterogeneity. Statistical heterogeneity between studies was explored using the $I^{2}$ statistics and Cochran's Q. $I^{2}>$ $25 \%, I^{2}>50 \%$, and $I^{2}>75 \%$ were regarded to indicate moderate, substantial, and considerable heterogeneity, respectively. ${ }^{23,29}$ A $P$ value $\leq 0.10$ for Cochran's $Q$ was regarded to indicate significant heterogeneity. ${ }^{23}$ Additionally $\tau^{2}$ was estimated to explore heterogeneity. While $I^{2}$ is interpreted as the percentage of variability in the treatment estimates, $\tau^{2}$ describes the underlying between-study variability. In contrast to the $I^{2}$ statistic, the $\tau^{2}$ estimate does not systematically increase with either the number of studies or the sample size. ${ }^{30}$

Risk of Bias Across Studies. Assessment of publication bias was originally planned by visual analysis of funnel plots. Because less than 10 studies were included, publication bias could not be assessed. ${ }^{31}$

\section{RESULTS}

\section{Literature Search}

A total of 121 records were retrieved in literature search. After eliminating duplicates 77 records remained, and 15 full-text articles were assessed for eligibility (Fig. 1). Finally, 6 RCTs with a total of 240 patients were eligible for qualitative analysis. Since one trials author ${ }^{32}$ did not provide adequate raw data of outcome measures upon request, only 5 RCTs were eligible for meta-analysis.] $\rightarrow$

\section{Study Characteristics}

Characteristics of the patient population, intervention, control condition, outcome measures, follow-ups, and results are shown in Table 1.

Setting and Patient Characteristics. All but one (Iran) ${ }^{33}$ of the included RCTs were conducted in India. Patients were recruited from neurology departments, ${ }^{32,33,36}$ medical research institutes, ${ }^{34,35}$ and private clinics ${ }^{37}$ and by newspaper advertisements. ${ }^{34}$ Patient's age in the included RCTs ranged from 15 to 60 years (mean $33.3 \pm 9.1$ years). Two RCTs included only patients suffering from TTH, ${ }^{32,35} 3$ RCTs included migraineurs only, ${ }^{33,34,36}$ and one RCT included patients with both headache types. ${ }^{37}$ Patients were diagnosed by neurologists, ${ }^{32-34,36}$ psychiatrists, ${ }^{35}$ and general physicians. ${ }^{37}$

Yoga Intervention. Two RCTs included yogic postures, breathing techniques, and relaxation in their intervention program. ${ }^{33,37}$ One RCTs program additionally comprised meditation. ${ }^{34}$ In three of the included studies, the program included either only postures, ${ }^{36}$ meditation, ${ }^{35}$ or relaxation. ${ }^{32}$ Five trials used hatha yoga as intervention, while one trial denoted their yoga as rajyoga. ${ }^{35}$ None of the included RCTs reported about daily homework. Program length reached from 6 weeks $^{36}$ to 16 weeks. ${ }^{37}$ Session duration reached from 30 $\min ^{32}$ to $75 \mathrm{~min}^{33}$ and frequency reached from one weekly session $^{35}$ to five weekly sessions. ${ }^{34,36}$

Control Conditions. Four RCTs compared yoga to usual care alone. ${ }^{33,35-37}$ One RCT compared yoga to a low-level self-care intervention in addition to usual care. ${ }^{34}$ While one RCT used EMG biofeedback additionally to usual care. ${ }^{32}$

Funding. Four of the six included studies reported on funding. ${ }^{32-34,36}$ Three studies were supported by grants of organizations that have no connections with the intervention. ${ }^{32-34}$ One study was supported by the Isfahan University of Medical Sciences and the Department of Exercise Physiology, University of Isfahan. ${ }^{33}$ One study received grants from the NMP Medical Research Institute, Jaipur, Rajasthan, India. ${ }^{34}$ One RCT was supported by the State Medical Research Council, Uttar Pradesh, India, ${ }^{32}$ whereas one study was supported by grants from the Central Council for Research in Yoga and Naturopathy, Department of AYUSH, Ministry of Health and Family Welfare, Government of India, New Delhi. ${ }^{36}$

\section{Risk of Bias}

Risk of bias for each study is shown in Table 2. While risk of selection bias was low in two of the included RCTs, ${ }^{33,34}$ two 


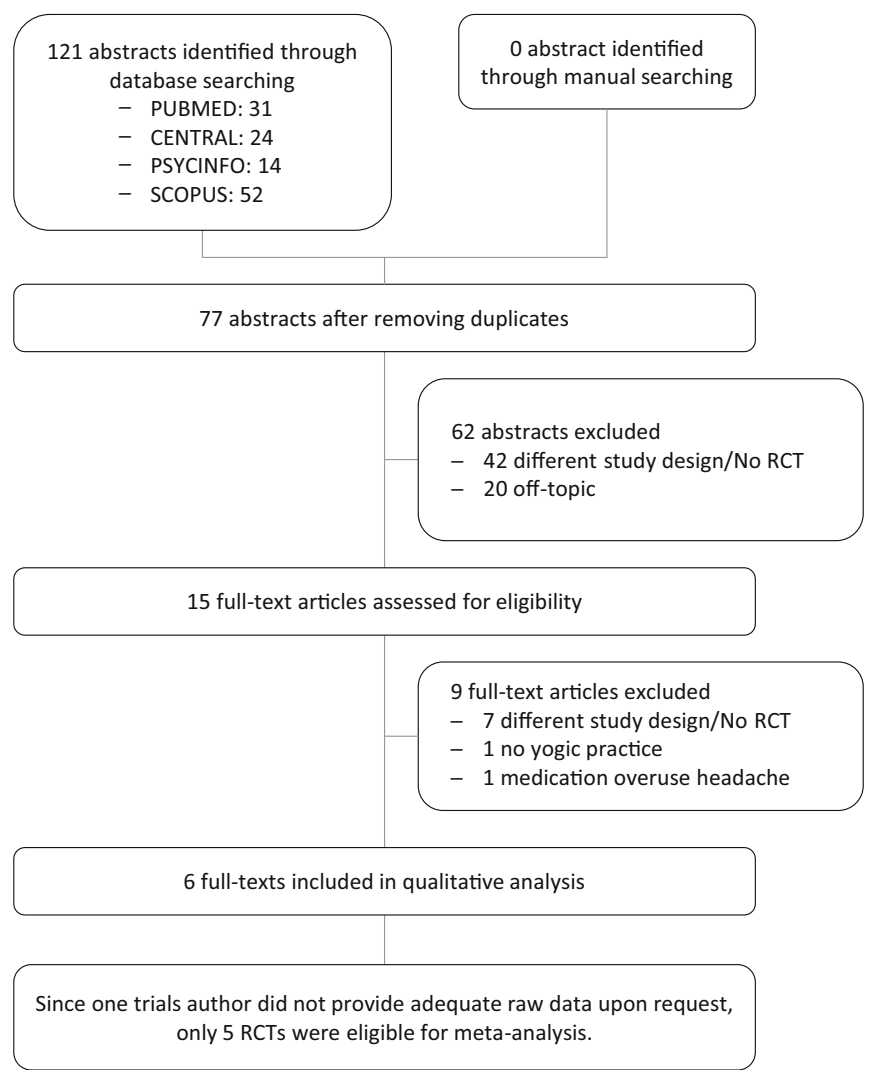

Figure 1 Flowchart of literature search.

RCTs did not report an adequate form of randomization..$^{35,37}$ In two studies, the random sequence generation remained unclear. ${ }^{32,36}$ None of the included RCTs did report an adequate form of allocation concealment. In four trials, risk of selection bias remained unclear, ${ }^{32-34,36}$ and two trials has to be judged as high risk studies. ${ }^{35,37}$ Two studies neither blinded outcome assessment nor participants and personnel. ${ }^{35,36}$ In four trials, performance and detection bias remained unclear. ${ }^{32-34,37}$ Risk of attrition bias was low in only one of the included studies, ${ }^{34}$ while in one study the risk was judged as unclear. ${ }^{37}$ Due to high drop-out rates and not performing intention-to-treat analyses, attrition bias was judged as high in the remaining four RCTs. ${ }^{32,33,35,36}$ Risk of reporting bias was low in four RCTs ${ }^{32,34-36}$ while in one study a high risk of selective reporting has to be suspected ${ }^{33}$ and in one trial such bias remained unclear. ${ }^{37}$ Due to inadequate statistical methods for all but one trial, ${ }^{34}$ a high risk of other bias has to be suspected.

\section{Assessment of Overall Effect Size}

Headache Frequency. All 6 RCTs assessed headache frequency post-intervention. While 5 trials assessed frequency by using headache diaries during intervention period, two trials used retrospective data. ${ }^{32,} 37$ One trial provided no additional raw data upon request and was therefore excluded from meta-analysis. ${ }^{32}$

Separate analysis by headache types revealed a statistical non-significant effect for migraine (3 RCTs; $\mathrm{SMD}=-1.96$;
$95 \% \mathrm{CI}-3.98$ to $0.05 ; I^{2}=80.0 \%, \tau^{2}=0.52, P<0.01$ ), while the effect for tension-type headache (1 RCT; SMD $=-2.25$; $95 \% \mathrm{CI}-2.98$ to 1.52 ) and mixed samples (1 RCT; SMD =$1.59 ; 95 \% \mathrm{CI}-2.63$ to -0.56 ) turned to be statistically significant.

Nevertheless, meta-analysis revealed a statistically significant overall effect for yoga in comparison to usual care at postintervention measurement (5 RCTs; SMD $=-1.97$; 95\% CI 2.75 to $-1.20 ; I^{2}=63.0 \%, \tau^{2}=0.25, P=0.03$ ) (Fig. 2).] $->$

Headache Duration. Headache duration was assessed by four RCT. ${ }^{33-35,37}$ All four studies used a headache diary to assess headache duration during intervention period.

No statistically significant effect could be detected for migraine if analyzed separately ( $2 \mathrm{RCTs}$; $\mathrm{SMD}=-1.03 ; 95 \% \mathrm{CI}$ -6.32 to $\left.4.27 ; I^{2}=69.0 \%, \tau^{2}=0.24, P=0.07\right)$, whereas the effects for tension-type headache (1 RCT; SMD $=2.18$; $95 \%$ $\mathrm{CI}-2.90$ to -1.46$)$ and mixed samples (1 RCT; SMD =$1.71 ; 95 \% \mathrm{CI}-2.77$ to -0.66$)$ turned to be statistically significant.

Also for headache duration, a statistically significant overall effect was found for yoga compared to usual care at postintervention ( $4 \mathrm{RCTs}$; SMD $=-1.45 ; 95 \% \mathrm{CI}-2.54$ to -0.37 ; $I^{2}=69.0 \%, \tau^{2}=0.33, P=0.02$ ) (Fig. 2).

Pain Intensity. Pain intensity was assessed by five of the included studies. ${ }^{33-37}$ While three RCTs used a $100-\mathrm{mm}$ visual analogue scale, ${ }^{33,35,36}$ one RCTs used an 11-point numeric 


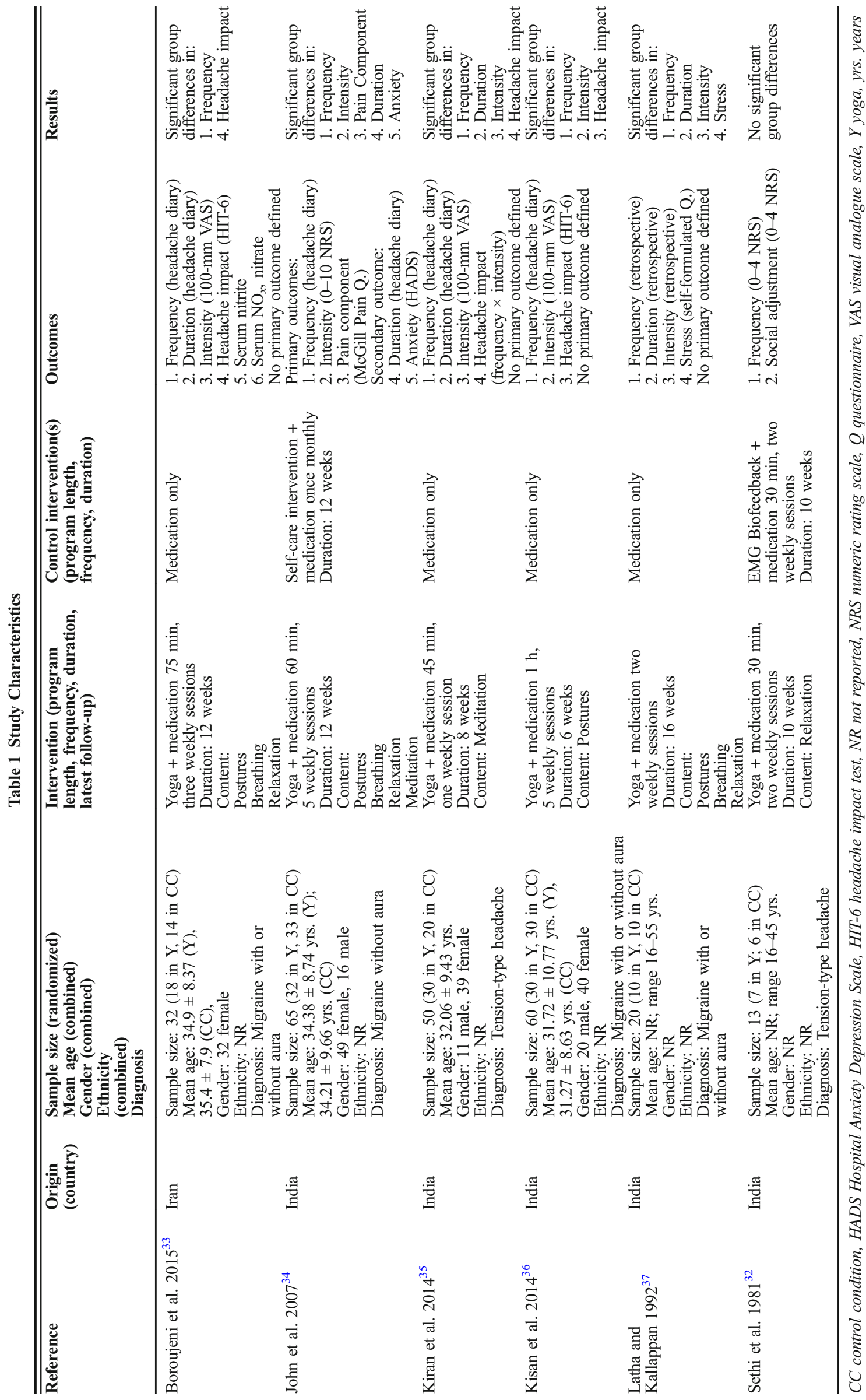


Table 2 Risk of Bias Assessment

\begin{tabular}{|c|c|c|c|c|c|c|c|}
\hline \multirow[t]{2}{*}{ Author, year } & \multicolumn{7}{|l|}{ Bias } \\
\hline & $\begin{array}{l}\text { Random } \\
\text { sequence } \\
\text { generation } \\
\text { (selection bias) }\end{array}$ & $\begin{array}{l}\text { Allocation } \\
\text { concealment } \\
\text { (selection bias) }\end{array}$ & $\begin{array}{l}\text { Blinding of } \\
\text { participants } \\
\text { and personnel } \\
\text { (performance } \\
\text { bias) }\end{array}$ & $\begin{array}{l}\text { Blinding of } \\
\text { outcome } \\
\text { assessment } \\
\text { (detection bias) }\end{array}$ & $\begin{array}{l}\text { Incomplete } \\
\text { outcome data } \\
\text { (attrition bias) }\end{array}$ & $\begin{array}{l}\text { Selective } \\
\text { reporting } \\
\text { (reporting } \\
\text { bias) }\end{array}$ & $\begin{array}{l}\text { Other } \\
\text { bias }\end{array}$ \\
\hline Boroujeni et al. $2015^{33}$ & Low risk & Unclear & Unclear & Unclear & High risk & High risk & $\overline{\text { High risk }}$ \\
\hline John et al. $2007^{34}$ & Low risk & Unclear & Unclear & Unclear & Low risk & Low risk & Unclear \\
\hline Kiran et al. $2014^{35}$ & High risk & High risk & High risk & High risk & High risk & Low risk & High risk \\
\hline Kisan et al. $2014^{36}$ & Unclear & Unclear & High risk & High risk & High risk & Low risk & High risk \\
\hline Latha and & High risk & High risk & Unclear & Unclear & Unclear & Unclear & High risk \\
\hline $\begin{array}{l}\text { Kallappan } 1992^{37} \\
\text { Sethi et al. } 1981^{32}\end{array}$ & Unclear & Unclear & Unclear & Unclear & High risk & Low risk & High risk \\
\hline
\end{tabular}

rating scale. ${ }^{34}$ Four RCTs assessed pain intensity via headache diary during intervention period. However, one study used retrospective data to assess pain intensity. ${ }^{37}$

If analyzed separately, no statistically significant effect was found for migraine ( $3 \mathrm{RCTs}$; SMD $=-3.00 ; 95 \% \mathrm{CI}-8.19$ to $\left.2.19 ; I^{2}=96.0 \%, \tau^{2}=4.20, P<0.01\right)$. However, a statistically significant effect was found for tension-type headache (1 RCT; SMD $=-6.15 ; 95 \% \mathrm{CI}-7.53$ to -4.77$)$ and mixed samples (1 RCT; SMD $=-2.14 ; 95 \% \mathrm{CI}-3.29$ to -0.99 ).

The overall effect for yoga in comparison to usual care at post-intervention turned also to be statistically

a Meta-analysis on headache frequency

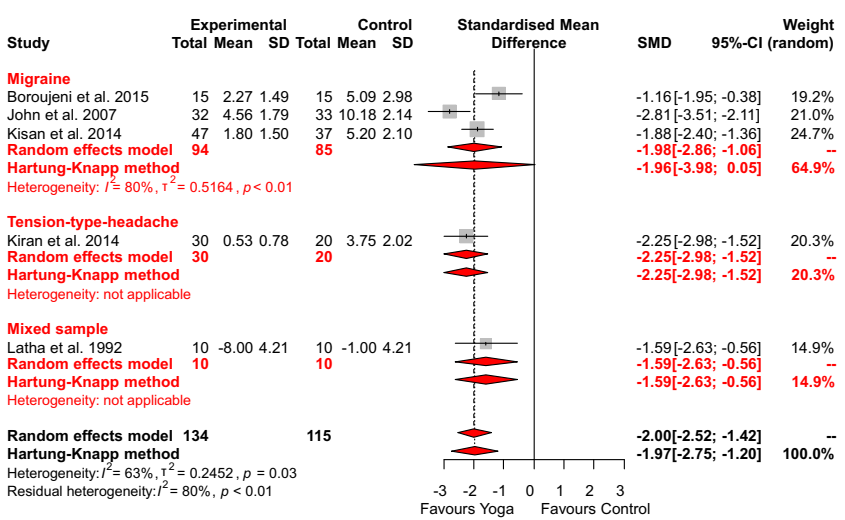

C Meta-analysis on pain intensity

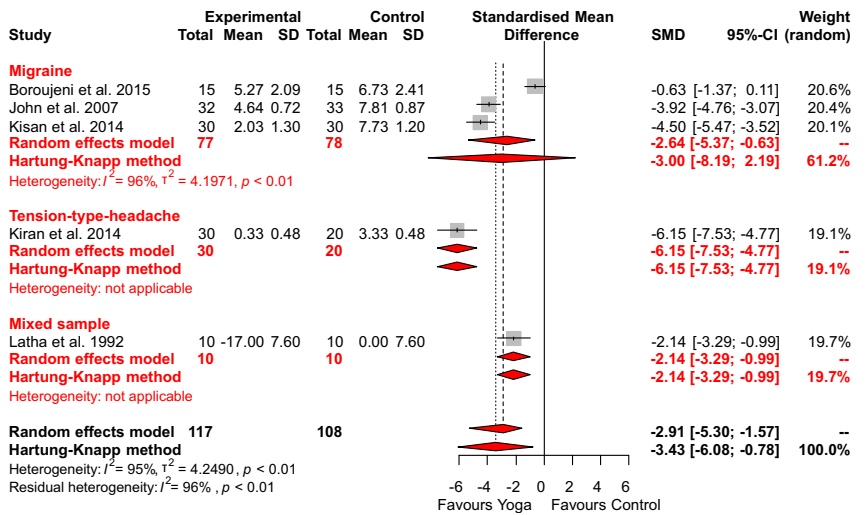

significant $(5$ RCTs; $\mathrm{SMD}=-3.43 ; 95 \% \mathrm{CI}-6.08$ to $-0.78 ; I^{2}=95.0 \%, \tau^{2}=4.25, P<0.01$ ) (Fig. 2).

Headache Impact. Three of the included trials assessed headache impact. Two RCTs used the headache impact test (HIT-6), ${ }^{33,36}$ while one study used a quotient of headache frequency and pain intensity. ${ }^{35}$

Also for headache impact, a separate analysis revealed a statistical non-significant effect for migraine (2 RCTs; $\mathrm{SMD}=-4.63 ; 95 \% \mathrm{CI}-48.70$ to $39.45 ; I^{2}=98.0 \%, \tau^{2}$ $=23.66, P<0.01)$. For tension-type headache, the effect

b Meta-analysis on headache duration

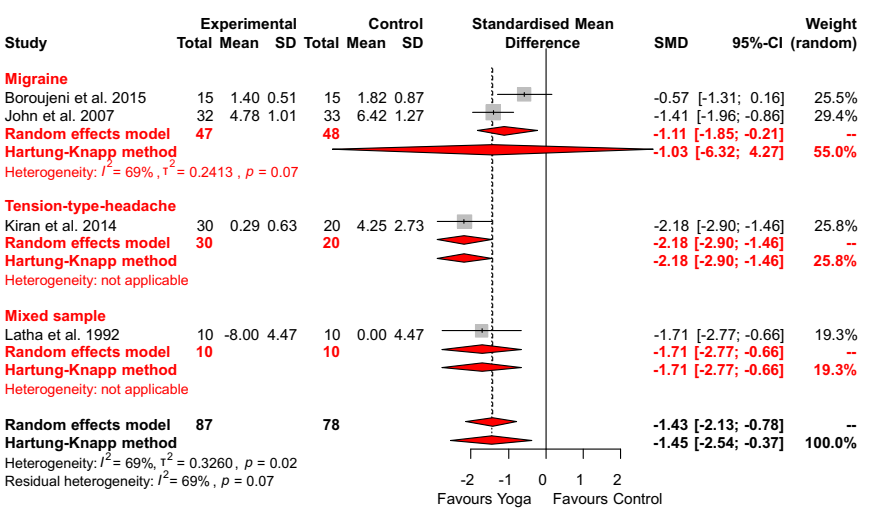

\section{d Meta-analysis on headache impact}

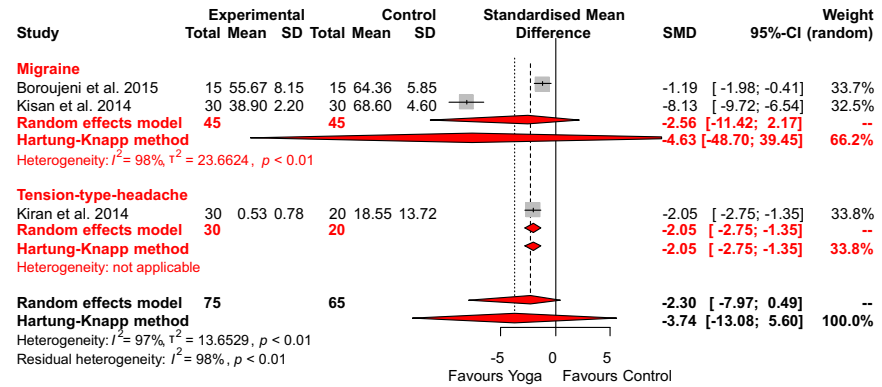

Figure 2 Meta-analysis of a headache frequency, b headache duration, c pain intensity, and d headache impact at post-intervention. 
turned to be statistically significant $(1 \mathrm{RCT}$; SMD = $2.05 ; 95 \%$ CI -2.75 to -1.35 ).

For headache impact, meta-analysis revealed no statistically significant overall effect at post-intervention (3 RCTs; SMD = $-3.74 ; 95 \%$ CI -13.08 to $5.60 ; I^{2}=97.0 \%, \tau^{2}=13.65, P<$ 0.01) (Fig. 2).

\section{Quality of Evidence}

For all measured outcomes and all separately analyzed headache types, the quality of evidence was downgraded to very low because of high likelihood of bias, serious limitations of the study quality, unexplained heterogeneity, and imprecision of results (Table 3 ).

\section{Safety and Adherence}

None of the included RCTs neither report occurrence (or absence) of adverse events nor discussed reasons for dropouts. Furthermore, none of the trials presented any data of adherence to the intervention or of practicing yoga after class.

\section{DISCUSSION}

\section{Summary of Evidence}

While overall effects revealed evidence that yoga can reduce headache frequency, headache duration, and pain intensity in chronic and episodic headache patients at post-intervention, the evaluation for the different headache types showed inconclusive evidence. For migraine patients, no statistically significant effects were detected for all outcomes. However, patients with tension-type headache reported significant effects for all observed outcomes, whereas no statistically significant effects for headache impact were found. It should be noted that the results for tension-type headache and mixed samples are based on one study only. Since none of the included studies performed follow-up assessments, no long-term effects could be detected.

In addition, the separate evaluation revealed significant differences between the two estimators used. While in the overall analysis the Knapp-Hartung estimator and the DerSimonian-Laird estimator differed only by the width of their confidence intervals, for migraine analysis the DerSimonian-Laird estimator produced statistically significant effects for headache frequency, headache duration, and pain intensity compared to the Knapp-Hartung estimator.

However, due to the low number of included studies, the relatively small overall population, high statistical heterogeneity, and the associated uncertainty in the parameter of estimation, the results of the Knapp-Hartung corrected estimator should be considered more reliable.

Since none of the included RCTs reported about occurrence (or absence) of adverse events as well as the attendance to the yoga class, no statement about safety and adherence can be made so far.

\section{Agreements with Prior Systematic Reviews}

To the best of our knowledge, this is the first meta-analysis to investigate the effects of yoga on chronic or episodic headaches. However, the results found coincide with other metaanalyses that have investigated the effects of yoga on various pain disorders. For example, a recently published Cochrane Review showed that yoga reduces the intensity of pain in patients with chronic low back pain if compared to standard treatment. ${ }^{12}$ Similar results were observed in patients with neck pain. ${ }^{38}$ Furthermore, yoga seems to be superior to nonmovement oriented mindfulness-based methods. For example, no pain-reducing effects, or only very low pain-reducing effects, had been demonstrated for MBSR in headaches and chronic low back pain. ${ }^{39,40}$

Table 3 Detailed Quality of Evidence Ratings According to GRADE

\begin{tabular}{|c|c|c|c|c|c|c|c|}
\hline Headache type & Outcome & $\begin{array}{l}\text { Limitations of the } \\
\text { study quality }\end{array}$ & Inconsistency & Indirectness & Imprecision & $\begin{array}{l}\text { Risk of } \\
\text { publication bias }\end{array}$ & $\begin{array}{l}\text { Quality of evidence } \\
\text { (GRADE) }\end{array}$ \\
\hline \multirow[t]{4}{*}{ TTH } & Frequency & Very serious & $\mathrm{n} / \mathrm{a}$ & None & Serious & $\mathrm{n} / \mathrm{a}$ & $\oplus \circ \circ \circ$ very low ${ }^{\dagger, *}$ \\
\hline & Duration & Very serious & $\mathrm{n} / \mathrm{a}$ & None & Serious & $\mathrm{n} / \mathrm{a}$ & $\bigoplus \circ 00$ very low ${ }^{\dagger,+}$ \\
\hline & Intensity & Very serious & $\mathrm{n} / \mathrm{a}$ & None & Serious & $\mathrm{n} / \mathrm{a}$ & $\bigoplus \circ \circ \circ$ very low ${ }^{\dagger,+}$ \\
\hline & Impact & Very serious & $\mathrm{n} / \mathrm{a}$ & None & Serious & $\mathrm{n} / \mathrm{a}$ & $\bigoplus \circ \circ \circ$ very low \\
\hline \multirow[t]{4}{*}{ Migraine } & Frequency & Very serious & Serious & None & Serious & $\mathrm{n} / \mathrm{a}$ & $\oplus \circ \circ \circ$ very low ${ }^{\dagger},+, \S$ \\
\hline & Duration & Very serious & Serious & None & Serious & $\mathrm{n} / \mathrm{a}$ & $\oplus \circ \circ \circ$ very low ${ }^{\dagger,},, \S$ \\
\hline & Intensity & Very serious & Serious & None & Serious & $\mathrm{n} / \mathrm{a}$ & $\oplus \circ \circ \circ$ very low ${ }^{\dagger,+, \S}$ \\
\hline & Impact & Very serious & Serious & None & Serious & $\mathrm{n} / \mathrm{a}$ & $\oplus \circ \circ \circ$ very low ${ }^{\dagger, \downarrow, \S}$ \\
\hline \multirow[t]{4}{*}{ Mixed sample } & Frequency & Very serious & $\mathrm{n} / \mathrm{a}$ & None & Serious & $\mathrm{n} / \mathrm{a}$ & $\bigoplus 00 \circ$ very low ${ }^{\dagger, 末}$ \\
\hline & Duration & Very serious & $\mathrm{n} / \mathrm{a}$ & None & Serious & $\mathrm{n} / \mathrm{a}$ & $\bigoplus 000$ very low ${ }^{\dagger, 末}$ \\
\hline & Intensity & Very serious & $\mathrm{n} / \mathrm{a}$ & None & Serious $\mathrm{s}$ & $\mathrm{n} / \mathrm{a}$ & $\bigoplus \circ \circ \circ$ very low ${ }^{\dagger,+}$ \\
\hline & Impact & Very serious & $\mathrm{n} / \mathrm{a}$ & None & Serious & $\mathrm{n} / \mathrm{a}$ & $\oplus \circ 00$ very low ${ }^{\dagger, \star}$ \\
\hline
\end{tabular}

$n / a=$ because only one study was included in meta-analysis

*Downgraded one level because of study limitations (overall unclear or high risk of bias)

†Downgraded two levels because of study limitations (overall unclear or high risk of bias)

tDowngraded one level because of imprecision (confidence interval includes negligible or no effects or fewer than 250 participants were included in total)

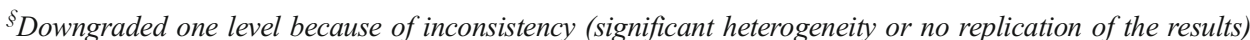

"Downgraded one level because of indirectness

\# Downgraded one level because of a probably high risk of publication bias 


\section{Limitations and Quality of Evidence}

However, the displayed results are afflicted by several limitations. Firstly, all included RCTs are from poor methodological quality. Only two trials reported about an adequate randomization, ${ }^{33,34}$ while in all RCTs allocation concealment has to be suspected as biased. Furthermore, all included trials used unsuitable statistical methods to evaluate between-group differences after a therapeutically intervention. Therefore, the quality of evidence was downgraded for all outcomes to very low.

Secondly, as all but one study were conducted in India the external validity of the results is limited. In this context, a recent overview found that RCTs on yoga that are conducted in India have about 25 times the odds of reaching positive conclusions as those conducted elsewhere. ${ }^{41}$ This may be due to the fact that yoga as an indigenous practice is part of the Indian spiritual and philosophical tradition and might thus induce larger unspecific effects than in other countries. Moreover, Indian yoga interventions often are much more intense than interventions in the western world.

Thirdly, none of the included RCTs reported about medication use. Different medication use among the participants might be a source for heterogeneity. Baseline differences must be taken into account, as well as a possible reduction in medication intake as a result of the treatment.

Fourthly, the study periods vary between the individual studies and thus also the time of the post-intervention measurement. Especially in the case of pain disorders and movement interventions, it cannot be ruled out that the influence of the intervention may increase over time. For this reason, it has to be expected that the effects of the included studies are influenced by their intervention length. This can further complicate the interpretation of the available results.

Fifthly, while all included studies describe that they adhered to the ICHD-III diagnostic guidelines when including patients, baseline study data indicate that both chronic and episodic headache patients were included. Although this has positive effects on the generalizability of the results, it in turn influences their heterogeneity and interpretability.

\section{Implications for Further Research}

Firstly, to determine long-term effects follow-up assessments with different time periods are urgently needed. Investigating long-term effects can help to uncover different progressions. This in turn may help to determine the optimal length of yoga interventions and the utility of practicing yoga at home.

Secondly, none of the included RCTs reported about adherence. To determine whether or not adherence and home practicing during intervention has an influence on the study effect, further trials should assess and report about both aspects.

Thirdly, also none of the included articles reported about occurrence (or absence) of adverse events. Since safety is crucial for the evaluation of therapies, future studies should address this aspect more accurate.
Fourthly, future RCTs should directly compare yoga with other mindfulness-based methods to investigate the potential benefits of yoga in the treatment of pain patients as described above.

\section{Conclusions}

Despite the aforementioned limitations, this systematic review found preliminary evidence of short-term efficacy of yoga in improving headache frequency, headache duration, and pain intensity, mainly in patients suffering from tension-type headaches. No effects were found for patients suffering from migraine. However, the low number of included studies, the relatively small overall population, high clinical and statistical heterogeneity, and the poor methodological quality of the included RCTs have to be taken into account and downgrade the quality of found evidence. Further high-quality trials with larger sample sizes and long-term measurements are needed before concrete recommendations for yoga in headaches can be made.

Role of the Funding Source: This review was supported by a grant from the Karl and Veronica-Carstens Foundation. The funding source had no influence on the design or conduct of the review; the collection, management, analysis, or interpretation of the data; or in the preparation, review, or approval of the manuscript. The authors have no financial relationships, related to this article, to disclose.

Corresponding Author: Dennis Anheyer, MA; Department of Internal and Integrative Medicine, Kliniken Essen-Mitte, Faculty of MedicineUniversity of Duisburg-Essen, Essen, Germany (e-mail: $d$. anheyer@kem-med.com).

Authors' Contributions DA was responsible for conception and design of the review, performed data extraction and analysis as well as assessment of risk of bias, and drafted the manuscript. HC and $P K$ carried out the literature search and performed data extraction and assessment of risk of bias, participated in the conception and design of the review, and critically revised the manuscript. RL and FJS participated in the conception and design of the review and critically revised the manuscript.

\section{Compliance with Ethical Standards:}

Conflict of Interest: All authors disclose any commercial association that might create a conflict of interest in connection with the submitted manuscript. There is especially no competing financial interest for any of the authors.

\section{REFERENCES}

1. Headache Classification Committee of the International Headache Society. The International Classification of Headache Disorders, 3rd edition (beta version). Cephalalgia. 2013;33:629-808.

2. Stovner $\mathbf{L}$, Hagen $\mathbf{K}$, Jensen $\mathbf{R}$, et al. The global burden of headache: a documentation of headache prevalence and disability worldwide. Cephalalgia. 2007;27:193-210.

3. Kessler RC, Shahly V, Stang PE, Lane MC. The associations of migraines and other headaches with work performance: results from the National Comorbidity Survey Replication (NCS-R). Cephalalgia. 2010; 30:722-34.

4. Steiner TJ, Stovner LJ, Vos T. Global Burden of Disease 2015: migraine is the third cause of disability in under 50s. J Headache Pain 2016;17:104.

5. Ayzenberg I, Katsarava Z, Sborowski A, Chernysh M, Osipova V, Tabeeva G, Steiner TJ. Headache-attributed burden and its impact on 
productivity and quality of life in Russia: structured healthcare for headache is urgently needed. Eur $\mathrm{J}$ Neurol 2014;21:758-65.

6. Cho SJ, Chu MK. Risk factors of chronic daily headache or chronic migraine. Curr Pain Headache Rep. 2015; 19:465.

7. Smitherman TA, Burch R, Sheikh H, Loder E. The prevalence, impact, and treatment of migraine and severe headaches in the United States: a review of statistics from national surveillance studies. Headache. 2013;53:427-36.

8. Thomas E, Boardman HF, Ogden H, Millson DS, Croft PR. Advice and care for headaches: who seeks it, who gives it? Cephalalgia. 2004;24:740 52.

9. Couch JR. Update on chronic daily headache. Curr Treat Options Neurol. 2011;13:41-55.

10. Houle T, Nash JM. Stress and headache chronification. Headache 2008;48:40-4.

11. Penzien DB, Irby MB, Smitherman TA, Rains JC, Houle TT. Wellestablished and empirically supported behavioral treatments for migraine. Curr Pain Headache Rep. 2015; 19:34.

12. Wieland LS, Skoetz $\mathbf{N}$, Pilkington $\mathbf{K}$, Vempati R, D'Adamo $\mathbf{C R}$ Berman BM. Yoga treatment for chronic non-specific low back pain. Cochrane Database Syst Rev. 2017;1:CD010671.

13. Bussing A, Ostermann T, Ludtke R, Michalsen A. Effects of yoga interventions on pain and pain-associated disability: a meta-analysis. $J$ Pain. 2012;13:1-9.

14. Michalsen A, Jeitler M, Brunnhuber S, et al. lyengar yoga for distressed women: a 3-armed randomized controlled trial. Evid Based Complement Alternat Med. 2012;2012:408727.

15. Cramer H, Lauche R, Anheyer D, Pilkington K, de Manincor M, Dobos G, Ward L. Yoga for anxiety: a systematic review and meta-analysis of randomized controlled trials. Depress Anxiety. 2018.

16. Cramer H, Anheyer D, Lauche R, Dobos G. A systematic review of yoga for major depressive disorder. J Affect Disord. 2017;213:70-7.

17. Cramer H, Lauche R, Langhorst J, Dobos G. Yoga for depression: a systematic review and meta-analysis. Depress Anxiety. 2013;30: 1068-83.

18. Lauche R, Langhorst J, Lee MS, Dobos G, Cramer H. A systematic review and meta-analysis on the effects of yoga on weight-related outcomes. Prev Med. 2016;87:213-32.

19. Cramer H, Ward L, Steel A, Lauche R, Dobos G, Zhang Y. Prevalence, patterns, and predictors of yoga use: results of a U.S. nationally representative survey. Am J Prev Med 2016;50:230-5.

20. Feuerstein G. The Yoga Tradition. Prescott: Hohm Press; 1998.

21. Iyengar BKS. Light on Yoga. New York: Schocken Books; 1966.

22. Moher D, Liberati A, Tetzlaff J, Altman DG. Preferred Reporting Items for Systematic Reviews and Meta-Analyses: the PRISMA statement. Ann Intern Med. 2009; 151:264-9, W64.

23. Higgins JPT, Green S. Cochrane Handbook for Systematic Reviews of Intervention.

24. Cornell JE, Mulrow CD, Localio R, Stack CB, Meibohm AR, Guallar E, Goodman SN. Random-effects meta-analysis of inconsistent effects: a time for change. Ann Intern Med. 2014;160:267-70.

25. Knapp G, Hartung J. Improved tests for a random effects metaregression with a single covariate. Stat Med. 2003;22:2693-710.
26. IntHout J, Ioannidis JP, Borm GF. The Hartung-Knapp-Sidik-Jonkman method for random effects meta-analysis is straightforward and considerably outperforms the standard DerSimonian-Laird method. BMC Med Res Methodol 2014;14:25.

27. Rover C, Knapp G, Friede T. Hartung-Knapp-Sidik-Jonkman approach and its modification for random-effects meta-analysis with few studies. BMC Med Res Methodol. 2015;15:99.

28. Cohen J. Statistical Power Analysis for the Behavioral Sciences. Hillsdale: Lawrence Erlbaum Associates; 1988.

29. Higgins JP, Thompson SG, Deeks JJ, Altman DG. Measuring inconsistency in meta-analyses. BMJ. 2003;327: 557-60.

30. Borenstein M, Hedges LV, Higgins JPT, Rothstein HR. Introduction to Meta-analysis. John Wiley \& Sons, Ltd; 2009.

31. Egger M, Davey Smith G, Schneider M, Minder C. Bias in metaanalysis detected by a simple, graphical test. BMJ 1997; 315: 629-34.

32. Sethi BB, Trivedi JK, Anand R. A comparative study of relative effectiveness of biofeedback and shavasana (yoga) in tension headache. Indian J Psychiatry. 1981;23:109-14.

33. Boroujeni MZ, Marandi SM, Esfarjani F, Sattar M, Shaygannejad V, Javanmard SH. Yoga intervention on blood NO in female migraineurs. Adv Biomed Res. 2015;4:259.

34. John PJ, Sharma N, Sharma CM, Kankane A. Effectiveness of yoga therapy in the treatment of migraine without aura: a randomized controlled trial. Headache. 2007;47:654-61.

35. Kiran, Girgla KK, Chalana $\mathbf{H}$, Singh $\mathbf{H}$. Effect of rajyoga meditation on chronic tension headache. Indian J Physiol Pharmacol. 2014;58.

36. Kisan $\mathbf{R}$, Sujan $\mathbf{M}$, Adoor $\mathbf{M}$, et al. Effect of yoga on migraine: a comprehensive study using clinical profile and cardiac autonomic functions. Int J Yoga. 2014;7:126-32.

37. Latha D, Kaliappan KV. Efficacy of yoga therapy in the management of headaches. J Indian Psychol. 1992;10:41-7.

38. Cramer H, Klose P, Brinkhaus B, Michalsen A, Dobos G. Effects of yoga on chronic neck pain: a systematic review and meta-analysis. Clin Rehabil. 2017;31:1457-65.

39. Anheyer D, Haller H, Barth J, Lauche R, Dobos G, Cramer H. Mindfulness-based stress reduction for treating low back pain: a systematic review and meta-analysis. Ann Intern Med. 2017;166:799807.

40. Anheyer D, Leach MJ, Klose P, Dobos G, Cramer H. Mindfulness-based stress reduction for treating chronic headache: a systematic review and meta-analysis. Cephalalgia. 2018;333102418781795.

41. Cramer H, Lauche R, Langhorst J, Dobos G. Are Indian yoga trials more likely to be positive than those from other countries? A systematic review of randomized controlled trials. Contemp Clin Trials. 2015;41:269-72.

Publisher's Note Springer Nature remains neutral with regard to jurisdictional claims in published maps and institutional affiliations. 